\title{
CONVEXITY PRESERVING FOR FULLY NONLINEAR PARABOLIC INTEGRO-DIFFERENTIAL EQUATIONS*
}

\author{
BAOJUN BIAN ${ }^{\dagger}$ AND PENGFEI GUAN ${ }^{\ddagger}$ \\ Dedicated to Professor Neil Trudinger on the occasion of his 65th birthday
}

Key words. Convexity preserving, constant rank principle, integro-differential equations.

AMS subject classifications. 35K10, 35B99

1. Introduction. In this paper, we consider the question of the preservation of convexity of the Cauchy problem for fully nonlinear integro-differential equation

$$
u_{t}=F\left(\nabla^{2} u, \nabla u, u, x, t\right)+\mathbb{B} u, \quad(x, t) \in \mathbb{R}^{n} \times[0, T],
$$

where $F=F(r, p, u, x, t)$ is a given function in $\Gamma=\mathcal{S}^{n} \times \mathbb{R}^{n} \times \mathbb{R} \times \mathbb{R}^{n} \times[0, T], \mathcal{S}^{n}$ denotes the space of real symmetric $n \times n$ matrices, and $\mathbb{B} u$ is a integro-differential operator as follows

$$
\mathbb{B} u=\lambda(t) \int_{0}^{1}(u(x+\psi(x, t, \eta), t)-u(x, t)-\psi(x, t, \eta) \cdot \nabla u(x, t)) d \eta,
$$

here $\lambda(t)$ is a given nonnegative function, $\psi(x, t, \eta)$ is a given function in $\mathbb{R}^{n} \times[0, T] \times$ $[0,1]$.

Equations (1.1) are second order fully nonlinear integro-differential equations of parabolic type. These equations are derived from the pricing problem of financial derivatives and optimal portfolio selection problem in a market where underlying assets prices are modeled by a Lévy process $S(\tau)$ (Chapter 9 in [14], see also [6], [16] and [3]). A typical example for the European option pricing problem in one-dimensional is as follows. Let $\left(W_{\tau}\right)_{\tau \geq 0}$ be the standard Brownian motion, $\left(N_{\tau}\right)_{\tau \geq 0}$ be Poisson process with parameter $\lambda$ and $\left(U_{j}\right)_{j \geq 1}$ be a sequence of square integrable independent, identically distributed random variables, with values in $(-1,+\infty)$. Assume the Lévy process $S(\tau)$ evolves according to the following stochastic differential equation

$$
\mathrm{d} S(\tau)=S(\tau)\left(\mu \mathrm{d} \tau+\sigma \mathrm{d} W_{\tau}+\mathrm{d}\left(\sum_{j=1}^{N_{\tau}} U_{j}\right)\right),
$$

where $\mu, \sigma$ are the drift and volatility respectively. Furthermore, we assume the processes $\left(W_{\tau}\right)_{\tau \geq 0},\left(N_{\tau}\right)_{\tau \geq 0},\left(U_{j}\right)_{j \geq 1}$ are independent. Let $\hat{p}(\xi)$ be the probability density function of random variable $U_{1}$, thus price function $V(s, \tau)$ of European option with finite horizon $T$ satisfies the following linear equation

$$
\frac{\partial V}{\partial \tau}+\frac{\sigma^{2}}{2} s^{2} \frac{\partial^{2} V}{\partial s^{2}}+(r-\lambda k) s \frac{\partial V}{\partial s}-(r+\lambda) V+\lambda \int_{-1}^{\infty} V(s(1+\xi), \tau) \hat{p}(\xi) d \xi=0
$$

\footnotetext{
*Received March 27, 2008; accepted for publication June 13, 2008.

${ }^{\dagger}$ Department of mathematics, Tongji University, Shanghai 200092, People's Republic of China (bianbj@mail.tongji.edu.cn). Research of the first author was supported in part by NSFC No.10671144 and National Basic Research Program of China (2007CB814903).

${ }^{\ddagger}$ Department of Mathematics and Statistics, McGill University, Montreal, Quebec, H3A 2K6, Canada (guan@math.mcgill.ca). Research of the second author was supported in part by an NSERC Discovery Grant.
} 
$(s, \tau) \in(0, \infty) \times[0, T)$, where $k=\int_{-1}^{\infty} \xi \hat{p}(\xi) d \xi$ and $V(s, T)=$ payoff. Moreover, if we change variables to $x=\ln s, t=T-\tau$ and $u(x, t)=V(s, \tau)$ we get

$$
\frac{\partial u}{\partial t}=\frac{\sigma^{2}}{2} \frac{\partial^{2} u}{\partial x^{2}}+\left(r-\lambda k-\frac{\sigma^{2}}{2}\right) \frac{\partial u}{\partial x}-(r+\lambda) u+\lambda \int_{-\infty}^{\infty} u(x+\eta, t) p(\eta) d \eta,
$$

$(x, t) \in \mathbb{R} \times(0, T]$, here $p(\eta)=e^{\eta} \hat{p}\left(e^{\eta}-1\right)$.

When we consider the option pricing with transaction costs and the optimal portfolio selection problem, the related equations become nonlinear equations, such as Hamilton-Jacobi-Bellman equations ([17], [2] and [3]).

The convexity preserving is connected to certain monotonicity properties of the option price with respect to volatility and other parameters in model ([7], [8] and references therein). This robustness properties motivates the study of convexity preserving. On the other hand, convexity of solution has important applications in the financial market completeness and super-replication ([15], [18]). We refer [11, 5, 4] for applications of convexity principles in differential geometry.

The problem of convexity preserving for solutions of linear equations has been studied extensively ([12], [7], [8] and references therein). In [12], S. Janson and J. Tysk obtained convexity preserving for linear parabolic differential equations. In [7] and [8], E. Ekstrom and J. Tysk discussed convexity preserving in the case of one spatial variable and several spatial variables for linear parabolic integro-differential equations. S. Lenhart [13] discussed the a-priori estimate and existence for strong solutions of linear parabolic integro-differential equations.

The main focus of this paper is to study the preservation of convexity of fully nonlinear parabolic integro-differential equation (1.1). We address both macro and micro convexity properties of solution to equation (1.1). We assume $F=F(r, p, u, x, t)$ is elliptic in the sense that

$$
\left(\frac{\partial F}{\partial r_{\alpha \beta}}\left(\nabla^{2} u, \nabla u, u, x, t\right)\right)>0, \quad \forall(x, t) \in \mathbb{R}^{n} \times[0, T] .
$$

Suppose the function $\psi=\psi(x, t, \eta) \in C^{2}\left(\mathbb{R}^{n}\right)$ and $\psi$ satisfies conditions

$$
\psi^{t} A \psi_{z z} \geq 0
$$

for any semi-positive definite matrix $A$ and for any unit direction $z \in \mathbb{R}^{n}$ (where $\psi^{t}$ is the transport of $\psi$ and $\left.\psi_{z z}=\psi_{x_{i} x_{j}} z_{i} z_{j}\right)$, and

$$
|\psi(x, t, \eta)| \leq M(1+|x|),\left|\nabla_{x} \psi(x, t, \eta)\right| \leq M,\left|\nabla_{x x}^{2} \psi(x, t, \eta)\right| \leq \frac{M}{1+|x|} .
$$

In this paper, we consider the Cauchy problem for equation (1.1) with given initial date $u(x, 0), x \in \mathbb{R}^{n}$. Define

$$
C_{p o l}=\cup_{m, M>0}\left\{u \in C\left(\mathbb{R}^{n} \times[0, T]\right)|| u(x, t) \mid \leq M\left(1+|x|^{m}\right)\right\} .
$$

We would like to find conditions on the functions $F(r, p, u, x, t)$ and $\psi(x, t, \eta)$ that guarantee that $C_{\text {pol }}$ solutions to the equation (1.1) remain convex if the initial condition is a convex function. Throughout this paper, we say $u(x, t)$ is convex means that $u(x, t)$ is a convex function in the spatial variable $x$ for each $t$ fixed.

We now state our main results. The first is of macro nature. 
Theorem 1.1. Assume (1.7) and (1.8) hold. Suppose the function $F=$ $F(r, p, u, x, t) \in C^{2,1}(\Gamma)$ satisfies conditions (1.6) and

$$
\begin{aligned}
& n=1, F(0, p, u, x, t) \quad \text { is locally convex in }(p, u, x) \text { for each } t \text { fixed, or } \\
& n \geq 2, F(r, p, u, x, t) \quad \text { is locally convex in }(r, p, u, x) \text { for each } t \text { fixed. }
\end{aligned}
$$

If $u \in C^{4,2}$ is a solution of equation (1.1), $\nabla^{2} u \in C_{\text {pol }}$, and there are constant $M$ such that

$$
\begin{aligned}
\left|F^{r_{i j}}\left(\nabla^{2} u, \nabla u, u, x, t\right)\right| & \leq M\left(1+|x|^{2}\right), \quad\left|F^{p_{k}}\left(\nabla^{2} u, \nabla u, u, x, t\right)\right| \leq M(1+|x|), \\
(1.10)^{u}\left(\nabla^{2} u, \nabla u, u, x, t\right) & \leq M .
\end{aligned}
$$

Then $u(x, t)$ is convex provided the initial date $u(x, 0)$ is convex.

The following is a micro-convexity result for solutions of equation (1.1). In this case, conditions in Theorem 1.1 can be relaxed.

Theorem 1.2. Suppose $F=F(r, p, u, x, t) \in C^{2,1}\left(\mathcal{S}^{n} \times \mathbb{R}^{n} \times \mathbb{R} \times \Omega \times(0, T)\right)$ for some domain $\Omega \subset \mathbb{R}^{n}$ and for some $T>0$, and suppose $F$ satisfies conditions (1.6), (1.9) and (1.7). Let $u \in C^{4,2}$ be a convex solution of equation (1.1), then at each time $t>0$, rank of $\nabla_{x}^{2} u(x, t)$ is constant. If let $l(t)=\min _{x \in \Omega}\left\{\right.$ rank of $\left.\nabla_{x}^{2} u(x, t)\right\}$, then $l(s) \leq l(t)$ for any $s \leq t<T$.

The rest of this paper is organized as follows. In section 2, we prove Theorem 1.2 following arguments in [4]. We prove Theorem 1.1 in section 3 . The last section is devoted to preservation of convexity of solutions for parabolic Bellman equations.

Acknowledgements. We would like to thank the referee for valuable suggestions.

2. A Constant Rank Theorem. In this section, we consider second order fully nonlinear differential equations of parabolic type

$$
u_{t}=F\left(\nabla^{2} u, \nabla u, u, x, t\right), \quad(x, t) \in \Omega \times[0, T],
$$

where $\Omega \subset \mathbb{R}^{n}$ is a domain. We assume $u \in C^{4,2}(\Omega \times[0, T])$ is a convex solution of equation (2.1) and will prove a constant rank theorem.

Let $W(x, t)=\nabla^{2} u(x, t)$ and $l=\min _{(x, t) \in \Omega \times[0, T]} \operatorname{rank}\left(\nabla^{2} u(x, t)\right)$. We may assume $l \leq n-1$. We define for $W=\left(u_{i j}\right) \in \mathcal{S}^{n}$

$$
q(W)=\left\{\begin{array}{lll}
\frac{\sigma_{l+2}(W)}{\sigma_{l+1}(W)}, & \text { if } & \sigma_{l+1}(W)>0 \\
0, & \text { if } & \sigma_{l+1}(W)=0
\end{array}\right.
$$

and

$$
\phi=\sigma_{l+1}(W)+q(W) .
$$

As in [4], we have

Proposition 2.1. Let $u \in C^{4,2}(\Omega \times[0, T])$ be a convex function and $W(x, t)=\left(u_{i j}(x, t)\right)$. Let $l=\min _{(x, t) \in \Omega \times[0, T]} \operatorname{rank}(W(x, t))$, then the function $q(x, t)=q(W(x, t))$ defined in (2.2) is in $C^{1+1,0+1}(\Omega \times[0, T])$. 
For any $n$, we denote $\mathcal{S}^{n}$ to be the set of all real symmetric $n \times n$ matrices, and denote $\mathcal{S}_{+}^{n} \subset \mathcal{S}^{n}$ to be the set of all positive definite symmetric $n \times n$ matrices. Let $\mathbb{O}_{n}$ be the space consisting all $n \times n$ orthogonal matrices. Let $0 \leq l \leq n$ we define

$$
\mathcal{S}_{l}=\left\{Q\left(\begin{array}{cc}
0 & 0 \\
0 & B
\end{array}\right) Q^{T} \quad \mid \quad \forall Q \in \mathbb{O}_{n}, \forall B \in \mathcal{S}^{l}\right\},
$$

and for given $Q \in \mathbb{O}_{n}$,

$$
\mathcal{S}_{l}(Q)=\left\{Q\left(\begin{array}{cc}
0 & 0 \\
0 & B
\end{array}\right) Q^{T} \quad \mid \quad \forall B \in \mathcal{S}^{l}\right\},
$$

therefore $\mathcal{S}_{l}, \mathcal{S}_{l}(Q) \subset \mathcal{S}^{n}$ and $\mathcal{S}_{0}=0, \mathcal{S}_{n}=\mathcal{S}^{n}$. For any function $F(r, p, u, x, t)$, we denote

$$
\begin{aligned}
& F^{\alpha \beta}=\frac{\partial F}{\partial r_{\alpha \beta}}, \quad F^{p_{k}}=\frac{\partial F}{\partial p_{k}}, \quad F^{u}=\frac{\partial F}{\partial u}, \quad F^{x_{i}}=\frac{\partial F}{\partial x_{i}}, \\
& F^{\alpha \beta, \gamma \eta}=\frac{\partial^{2} F}{\partial r_{\alpha \beta} \partial r_{\gamma \eta}}, \quad F^{\alpha \beta, p_{k}}=\frac{\partial^{2} F}{\partial r_{\alpha \beta} \partial p_{k}}, \quad F^{\alpha \beta, u}=\frac{\partial^{2} F}{\partial r_{\alpha \beta} \partial u}, \\
& F^{\alpha \beta, x_{k}}=\frac{\partial^{2} F}{\partial r_{\alpha \beta} \partial x_{k}}, \quad F^{p_{k}, p_{l}}=\frac{\partial^{2} F}{\partial p_{k} \partial p_{l}}, \quad F^{p_{k}, u}=\frac{\partial^{2} F}{\partial p_{k} \partial u}, \\
& F^{p_{k}, x_{j}}=\frac{\partial^{2} F}{\partial p_{k} \partial x_{j}}, \quad F^{u, u}=\frac{\partial^{2} F}{\partial^{2} u}, \quad F^{u, x_{i}}=\frac{\partial^{2} F}{\partial u \partial x_{i}}, \quad F^{x_{i}, x_{j}}=\frac{\partial^{2} F}{\partial x_{i} \partial x_{j}} .
\end{aligned}
$$

Let $0 \leq l \leq n-1, B \in \mathcal{S}_{+}^{l}, A=B^{-1}$ and

$$
\tilde{B}=\left(\begin{array}{cc}
0 & 0 \\
0 & B
\end{array}\right), \quad \tilde{A}=\left(\begin{array}{cc}
0 & 0 \\
0 & A
\end{array}\right)
$$

For any fixed $Q \in \mathbb{O}_{n}$ and $\tilde{X}=(X, Y, z) \in \mathcal{S}_{l}(Q) \times \mathbb{R} \times \mathbb{R}^{n}$, we define a quadratic form

$$
\begin{aligned}
Q^{*}(\tilde{X}, \tilde{X})= & \sum_{i, j, k, l=1}^{n}\left[F^{i j, k l} X_{i j} X_{k l}+2 F^{i j}\left(Q \tilde{A} Q^{T}\right)_{k l} X_{i k} X_{j l}\right]-2 \sum_{i, j=1}^{n} F^{i j, u} X_{i j} Y \\
& -2 \sum_{i, j, k=1}^{n} F^{i j, x_{k}} X_{i j} z_{k}+F^{u, u} Y^{2}+2 \sum_{i=1}^{n} F^{u, x_{i}} Y z_{i}+\sum_{i, j=1}^{n} F^{x_{i}, x_{j}} z_{i} z_{j},
\end{aligned}
$$

where $\quad F^{i j, k l}, F^{i j}, F^{u, u}, F^{i j, u}, F^{i j, x_{k}}, F^{u, x_{i}}, F^{x_{i}, x_{j}} \quad$ are evaluated at $\left(Q \tilde{B} Q^{T}, \nabla u(x, t), u, x, t\right)$. We assume

$$
Q^{*}=Q^{*}\left(\tilde{X}^{m}, \tilde{X}^{m}\right) \geq 0, \quad m=1, \cdots, n-l,
$$

for any fixed $0 \leq l \leq n-1, \tilde{B}, \tilde{A}, Q, \tilde{X^{m}}=\left(X, Y, z^{m}\right) \in \mathcal{S}_{l}(Q) \times \mathbb{R} \times \mathbb{R}^{n}$ and $z^{m}=\left(Q_{1 m}, \cdots, Q_{n m}\right)$.

Remark 2.2. Condition (2.6) is weaker than condition (1.9). In particular (2.6) is empty condition in $r$ when $n=1$.

TheOrem 2.3. Suppose $F=F(r, p, u, x, t) \in C^{2,1}(\Gamma)$ and $F$ satisfies conditions (1.6) and (2.6). Let $u \in C^{4,2}$ be a convex solution of equation (2.1) in $\Omega \times[0, T)$. 
For each $T>t>0$, let $l(t)$ be the minimal rank of $\nabla^{2} u(x, t)$ in $\Omega$. Then, the rank of $\nabla^{2} u(x, t)$ is constant for each $T>t>0$ and $l(s) \leq l(t)$ for all $s \leq t<T$.

Proof. The proof is similar to the proof of Theorem 3.2 in [4] with some minor modifications.

Let $u \in C^{4,2}$ be a convex solution of equation $(2.1)$ and $W(x, t)=\left(u_{i j}(x, t)\right)$. For each $t_{0}>0$, suppose $W=\nabla^{2} u\left(x, t_{0}\right)$ attains minimal rank $l$ at some point $x_{0} \in \Omega$. We may assume $l \leq n-1$, otherwise there is nothing to prove. By continuity, $\sigma_{l}\left(u_{i j}(x, t)\right)>0$ in a neighborhood of $\left(x_{0}, t_{0}\right)$. As in [4], we pick an open neighborhood $\mathcal{O} \subset \Omega \times[0, T)$ of $\left(x_{0}, t_{0}\right)$, for any $(x, t) \in \mathcal{O}$, let $G=\{n-l+1, n-l+2, \ldots, n\}$ and $B=\{1, \ldots, n-l\}$ be the "good" and "bad" sets of indices for eigenvalues of $\nabla^{2} u(x, t)$ respectively.

Setting $\phi$ as $(2.3)$, then we see from Proposition $(2.1)$ that $\phi \in C^{1+1,0+1}(\mathcal{O})$,

$$
\phi(x, t) \geq 0, \phi\left(x_{0}, t_{0}\right)=0
$$

and there is a constant $C>0$ such that for all $(x, t) \in \mathcal{O}$,

$$
\begin{gathered}
\frac{1}{C} \sigma_{1}(B)(x, t) \leq \sigma_{l+1}(W)(x, t) \leq C \sigma_{1}(B)(x, t), \\
\frac{1}{C} \sigma_{1}(B)(x, t) \leq \phi(x, t) \leq C \sigma_{1}(B)(x, t) .
\end{gathered}
$$

We will derive the following differential inequality (2.7) for $\phi$. Then this theorem follows from the strong maximum principle.

$$
\sum F^{\alpha \beta} \phi_{\alpha \beta}(x, t)-\phi_{t}(x, t) \leq C_{1} \phi(x, t)+C_{2}|\nabla \phi(x, t)| \text {. }
$$

We shall fix a point $(y, \tau) \in \mathcal{O}$ and prove inequality $(2.7)$ at $(y, \tau)$. For each $(y, \tau) \in \mathcal{O}$ fixed, letting $\lambda_{1} \leq \lambda_{2} \ldots \leq \lambda_{n}$ be the eigenvalues of $W(y, \tau)=\left(u_{i j}(y, \tau)\right)$, we can rotate coordinate so that $W(y, \tau)=\left(u_{i j}(y, \tau)\right)$ is diagonal, and $u_{i i}(y, \tau)=$ $\lambda_{i}, i=1, \cdots, n$. We note that all quantities involving $g, q$ and $\phi$ are invariant under rotation.

Again, as in [4], we will avoid to deal with $\sigma_{l+1}(W)=0$ by considering for $W_{\epsilon}$ for $\epsilon>0$ sufficient small, with $W_{\epsilon}=W+\epsilon I, G_{\epsilon}=\left(\lambda_{n-l+1}+\epsilon, \ldots, \lambda_{n}+\epsilon\right)$, $B_{\epsilon}=\left(\lambda_{1}+\epsilon, \ldots, \lambda_{n-1}+\epsilon\right)$. We note that $W_{\epsilon}$ is the Hessian of function $u_{\epsilon}(x, t)=$ $u(x, t)+\frac{\epsilon}{2}|x|^{2}$. This function $u_{\epsilon}(x, t)$ satisfies equation

$$
\left(u_{\epsilon}\right)_{t}-F\left(\nabla^{2} u_{\epsilon}, \nabla u_{\epsilon}, u_{\epsilon}, x, t\right)=-R_{\epsilon},
$$

where $R_{\epsilon}=R_{\epsilon}(x, t)=F\left(\nabla^{2} u_{\epsilon}, \nabla u_{\epsilon}, u_{\epsilon}, x, t\right)-F\left(\nabla^{2} u, \nabla u, u, x, t\right)$. Since $u \in C^{4,2}$, we have

$$
\left|R_{\epsilon}\right| \leq C \epsilon, \quad\left|\nabla R_{\epsilon}\right| \leq C \epsilon, \quad\left|\nabla^{2} R_{\epsilon}\right| \leq C \epsilon, \quad \forall(x, t) \in \mathcal{O}
$$

We will work on equation (2.8) to obtain a differential inequality for $\phi_{\epsilon}$

$$
\sum F^{\alpha \beta} \phi_{\alpha \beta}(y, \tau)-\phi_{t}(y, \tau) \leq C_{1} \phi(y, \tau)+C_{2}|\nabla \phi(y, \tau)|+O(\epsilon) .
$$

The differential inequality (2.7) will follow from (2.10) by letting $\epsilon \rightarrow 0$. Set $v=u_{\epsilon}$, in the rest of this section, we will write $q$ for $q_{\epsilon}, W$ for $W_{\epsilon}, G$ for $G_{\epsilon}$ and $B$ for $B_{\epsilon}$ 
with the understanding that all the estimates will be independent of $\epsilon$. We note that $v$ satisfies equation (2.8).

We differentiate equation (2.8) in $x_{i}$, by (2.9), thereby obtaining equation

$$
\sum_{\alpha \beta} F^{\alpha \beta} v_{\alpha \beta i}+\sum_{k} F^{p_{k}} v_{k i}+F^{v} v_{i}+F^{x_{i}}=v_{i, t}+O(\epsilon)
$$

and differentiate equation (2.8) twice with respect to the variables $x_{i}$ and $x_{j}$, we have

$$
\begin{aligned}
& \sum_{\alpha \beta} F^{\alpha \beta} v_{\alpha \beta i j}+\sum_{\alpha \beta} v_{\alpha \beta i}\left(\sum_{\gamma \eta} F^{\alpha \beta, \gamma \eta} v_{\gamma \eta j}+\sum_{k} F^{\alpha \beta, p_{k}} v_{k j}+F^{\alpha \beta, v} v_{j}+F^{\alpha \beta, x_{j}}\right) \\
& +\sum_{k} F^{p_{k}} v_{k i j}+\sum_{k} v_{k i}\left(\sum_{\alpha \beta} F^{p_{k}, \alpha \beta} v_{\alpha \beta j}+\sum_{l} F^{p_{k}, p_{l}} v_{l j}+F^{p_{k}, v} v_{j}+F^{p_{k}, x_{j}}\right) \\
& +F^{v} v_{i j}+v_{i}\left(\sum_{\alpha \beta} F^{v, \alpha \beta} v_{\alpha \beta j}+\sum_{l} F^{v, p_{l}} v_{l j}+F^{v, v} v_{j}+F^{v, x_{j}}\right) \\
& \quad+\sum_{\alpha \beta} F^{x_{i}, \alpha \beta} v_{\alpha \beta j}+\sum_{k} F^{x_{i}, p_{k}} v_{k j}+F^{x_{i}, v} v_{j}+F^{x_{i}, x_{j}}=v_{i j, t}+O(\epsilon) .
\end{aligned}
$$

A simple computation yields

$$
\begin{gathered}
\phi_{\alpha}=\frac{\partial \phi}{\partial x_{\alpha}}=\phi^{i j} v_{i j \alpha} \\
\phi_{\alpha \beta}=\frac{\partial^{2} \phi}{\partial x_{\alpha} \partial x_{\beta}}=\phi^{i j} v_{i j \alpha \beta}+\phi^{i j, k m} v_{i j \alpha} v_{k m \beta},
\end{gathered}
$$

and accordingly

$$
\begin{aligned}
\sum F^{\alpha \beta} \phi_{\alpha \beta}= & \sum F^{\alpha \beta} \phi^{i j} v_{i j \alpha \beta}+\sum F^{\alpha \beta} \phi^{i j, k m} v_{i j \alpha} v_{k m \beta} \\
= & \sum F^{\alpha \beta} \phi^{i j, k m} v_{i j \alpha} v_{k m \beta}-\sum \phi^{i j} F^{p_{k}} v_{k i j} \\
& -\sum \phi^{i j}\left[F^{v} v_{i j}+2 \sum F^{\alpha \beta, p_{k}} v_{\alpha \beta i} v_{k j}+\sum F^{p_{k}, p_{l}} v_{k i} v_{l j}\right. \\
& \left.+2 \sum F^{p_{k}, v} v_{k i} v_{j}+2 \sum F^{p_{k}, x_{j}} v_{k i}\right] \\
& -\sum \phi^{i j}\left[F^{\alpha \beta, \gamma \eta} v_{\alpha \beta i} v_{\gamma \eta j}+2 \sum F^{\alpha \beta, v} v_{\alpha \beta i} v_{j}+2 \sum F^{\alpha \beta, x_{j}} v_{\alpha \beta i}\right. \\
& \left.+\sum F^{v, v} v_{i} v_{j}+2 \sum F^{v, x_{j}} v_{j}+\sum F^{x_{i} x_{j}}\right]+O(\epsilon)+\sum \phi^{i j} v_{i j, t} .
\end{aligned}
$$

We note that $\phi_{t}=\sum \phi^{i j} v_{i j, t}$, equation (2.13) can be written as

$$
\begin{aligned}
\sum F^{\alpha \beta} \phi_{\alpha \beta}-\phi_{t}= & \sum F^{\alpha \beta} \phi^{i j, k m} v_{i j \alpha} v_{k m \beta}-\sum \phi^{i j} F^{p_{k}} v_{k i j} \\
& -\sum \phi^{i j}\left[F^{v} v_{i j}+2 \sum F^{\alpha \beta, p_{k}} v_{\alpha \beta i} v_{k j}+\sum F^{p_{k}, p_{l}} v_{k i} v_{l j}\right. \\
& \left.+2 \sum F^{p_{k}, v} v_{k i} v_{j}+2 \sum F^{p_{k}, x_{j}} v_{k i}\right] \\
& -\sum \phi^{i j}\left[F^{\alpha \beta, \gamma \eta} v_{\alpha \beta i} v_{\gamma \eta j}+2 \sum F^{\alpha \beta, v} v_{\alpha \beta i} v_{j}+2 \sum F^{\alpha \beta, x_{j}} v_{\alpha \beta i}\right. \\
& \left.+\sum F^{v, v} v_{i} v_{j}+2 \sum F^{v, x_{j}} v_{j}+\sum F^{x_{i} x_{j}}\right]+O(\epsilon) .
\end{aligned}
$$


We estimate for $i \in B$

$$
\begin{aligned}
& J_{i}=\left[\sum_{\alpha, \beta, \gamma, \eta \in G} F^{\alpha \beta, \gamma \eta} v_{i \alpha \beta} v_{i \gamma \eta}+2 \sum_{\alpha, \beta \in G} F^{\alpha \beta} \sum_{j \in G} \frac{1}{\lambda_{j}} v_{i j \alpha} v_{i j \beta}\right. \\
& \left.\quad+2 \sum_{\alpha, \beta \in G} F^{\alpha \beta, v} v_{i \alpha \beta} v_{i}+2 \sum_{\alpha, \beta \in G} F^{\alpha \beta, x_{i}} v_{i \alpha \beta}+F^{v, v} v_{i}^{2}+2 F^{v, x_{i}} v_{i}+F^{x_{i}, x_{i}}\right],
\end{aligned}
$$

where functions $F^{i j, k l}, F^{i j}, F^{u, u}, F^{i j, u}, F^{i j, x_{k}}, F^{u, x_{i}}, F^{x_{i}, x_{j}}$ are evaluated at

$$
\left(\nabla^{2} v(y, \tau), \nabla v(y, \tau), v(y, \tau), y, \tau\right)
$$

Since $F \in C^{2,1}$ and $v_{i j}=\delta_{i j} \lambda_{i}$ at $(y, \tau)$, we conclude that

$$
\begin{aligned}
J_{i}= & {\left[\sum_{\alpha, \beta, \gamma, \eta \in G} F^{\alpha \beta, \gamma \eta} v_{i \alpha \beta} v_{i \gamma \eta}+2 \sum_{\alpha, \beta, \gamma, \eta \in G} F^{\alpha \beta} \tilde{A}_{\gamma \eta} v_{i \alpha \gamma} v_{i \beta \eta}+2 \sum_{\alpha, \beta \in G} F^{\alpha \beta, v} v_{i \alpha \beta} v_{i}\right.} \\
& \left.+2 \sum_{\alpha, \beta \in G} F^{\alpha \beta, x_{i}} v_{i \alpha \beta}+F^{v, v} v_{i}^{2}+2 F^{v, x_{i}} v_{i}+F^{x_{i}, x_{i}}\right]+O(\phi),
\end{aligned}
$$

where $\quad F^{i j, k l}, F^{i j}, F^{u, u}, F^{i j, u}, F^{i j, x_{k}}, F^{u, x_{i}}, F^{x_{i}, x_{j}} \quad$ are evaluated at $(\tilde{B}, \nabla v(y, \tau), v(y, \tau), y, \tau)$ and $\tilde{B}=\operatorname{diag}\left(0, \cdots, 0, \lambda_{n-l+1}, \cdots, \lambda_{n}\right)$. Now let's set $X_{\alpha \beta}=0, \alpha \in B$ or $\beta \in B, X_{\alpha \beta}=v_{i \alpha \beta}$ otherwise, $Y=-v_{i}$ and $z_{k}=-\delta_{k i}$. Then $\left(X_{\alpha \beta}\right) \in \mathcal{S}_{l}$ (identity matrix). Noting all quantities are under a rotation $Q$. By condition (2.6), we infer that

$$
J_{i} \geq-C \phi
$$

Now the same argument in the proof of Theorem 3.2 in [4] yields (2.10). Taking $\epsilon \rightarrow 0$ and applying the strong maximum principle for parabolic equation, we conclude that $\phi$ vanishes everywhere $\Omega \times\left[0, t_{0}\right]$. In particular, $\sigma_{l+1}\left(u_{i j}(x, t)\right) \equiv 0$ in $\Omega \times\left[0, t_{0}\right]$.

口

Let us come back to prove Theorem 1.2.

Proof of Theorem 1.2. Let

$$
\tilde{F}(r, p, u, x, t)=F(r, p, u, x, t)+\lambda(t) \int_{0}^{1}(u(x+\psi(x, t, \eta), t)-u-\psi(x, t, \eta) \cdot p) d \eta
$$

then $\tilde{F}$ satisfies conditions conditions (1.6) and (2.6) whenever (1.7) holds and $u(x, t)$ is a convex function. $\square$

3. Convexity Preserving for Fully Nonlinear Parabolic Integrodifferential Equations. We prove Theorem 1.1 in this section. Since we are dealing the problem in the whole ambient space $\mathbb{R}^{n}$, we need certain growth conditions specified in Theorem 1.1 to construct a barrier to gain control at $\infty$.

Proof of Theorem 1.1. Suppose $u \in C^{4,2}\left(\mathbb{R}^{n} \times[0, T]\right)$ is a solution of equation (1.1), $\nabla^{2} u \in C_{\text {pol }}$. Let $\mathrm{m}$ be an positive constant chosen so large that $\left|\nabla^{2} u(x, t)\right|=$ $O\left(1+|x|^{m}\right)$. Set $g(x)=\left(1+|x|^{2}\right)^{\frac{m}{2}+1+\alpha}, \alpha>0$ and $w(x, t)=e^{K t} g(x)$. Let $\epsilon>0$ and define

$$
u^{\epsilon}(x, t)=u(x, t)+\epsilon w(x, t)
$$


Let $\lambda(x, t)=\lambda_{\epsilon}(x, t)$ be the smallest eigenvalue of $\nabla^{2} u^{\epsilon}(x, t)$ and define

$$
E=\left\{(x, t) \in \mathbb{R}^{n} \times[0, T] \mid \lambda(x, t) \leq 0\right\} .
$$

Suppose that $E \neq \emptyset$. Simple computation yields

$$
\nabla^{2} g(x) \geq\left(\left(1+|x|^{2}\right)^{\frac{m}{2}+\alpha} \delta_{i j}\right)
$$

therefore

$$
\lambda(x, t) \geq \epsilon e^{K t}\left(1+|x|^{2}\right)^{\frac{m}{2}+\alpha}-O\left(1+|x|^{m}\right) .
$$

Hence there is a constant $R=R_{\epsilon}$ such that $\lambda(x, t)>0$ for $|x| \geq R$. Thus $E \subseteq$ $B_{R} \times[0, T]$ and is compact. Let

$$
t_{0}=\min \left\{t \geq 0 \mid(x, t) \in E \text { for some } x \in \mathbb{R}^{n}\right\} .
$$

This minimum is attained and thus $\left(x_{0}, t_{0}\right) \in E$ and $\lambda\left(x_{0}, t_{0}\right) \leq 0$ for some $x_{0} \in B_{R}$.

We have $\nabla^{2} u^{\epsilon}(x, 0) \geq \epsilon \nabla^{2} g(x)>0$ from the convexity of $u(x, 0)$, hence $t_{0}>0$ by the continuity. Since $\lambda(x, t)>0$ for all $0<t<t_{0}$ and $x \in \mathbb{R}^{n}, \lambda\left(x, t_{0}\right) \geq 0$ for all $x \in \mathbb{R}^{n}$ by the continuity. Consequently, $u^{\epsilon}(x, t)$ is convex in $B_{R} \times\left[0, t_{0}\right]$ but $\lambda\left(x_{0}, t_{0}\right)=0$. Hence the Hessian $\nabla^{2} u^{\epsilon}(x, t)$ attains minimum rank $l \leq n-1$ at some point $\left(x_{1}, t_{0}\right)$ for some $x_{1} \in B_{R}$.

Function $u^{\epsilon}(x, t)$ satisfies equation

$$
u_{t}^{\epsilon}=F_{\epsilon}\left(\nabla^{2} u^{\epsilon}, \nabla u^{\epsilon}, u^{\epsilon}, x, t\right)+\mathbb{B} u^{\epsilon},
$$

where

$$
F_{\epsilon}(r, p, u, x, t)=F\left(r-\epsilon \nabla^{2} w, p-\epsilon \nabla w, u-\epsilon w, x, t\right)+\epsilon\left(w_{t}-\mathbb{B} w\right) .
$$

We need the following lemma.

Lemma 3.1. Assume conditions (1.8), (1.6), (1.9) and (1.10) hold. Then we can choose constant $K$ such that $F_{\epsilon}$ satisfies conditions (1.6) and (2.6).

If this lemma is true, applying Theorem 1.2, we deduce that the rank of Hessian $\nabla^{2} u^{\epsilon}(x, t)$ is constant in $B_{R} \times\left(0, t_{0}\right]$. This is a contradiction and hence $E=\emptyset$. Therefore $\nabla^{2} u^{\epsilon}(x, t)>0$ for all $(x, t) \in \mathbb{R}^{n} \times[0, T]$ and this implies the convexity of $u(x, t)$. Therefore, we need to verify Lemma 3.1.

Proof of Lemma 3.1. It is clear that $F_{\epsilon}$ is elliptic. The remainder is to check condition (2.6) for $F_{\epsilon}$ and any given $0 \leq l \leq n-1, \tilde{B}, \tilde{A}, Q \in \mathbb{O}_{n}, \tilde{X}=(X, Y, z) \in$ $\mathcal{S}_{l}(Q) \times \mathbb{R} \times \mathbb{R}^{n}$ and $z=z^{m}=\left(Q_{1 m}, \cdots, Q_{n m}\right)$ for all $m=1, \cdots, n-l$. A direct computation yields

$$
\begin{gathered}
F_{\epsilon}^{i j}(r, p, u, x, t)=F^{i j}\left(r-\epsilon \nabla^{2} w, p-\epsilon \nabla w, u-\epsilon w, x, t\right), \quad F_{\epsilon}^{u}=F^{u}, \\
F_{\epsilon}^{x_{k}}=F^{i j}\left(-\epsilon w_{k i j}\right)+F^{p_{i}}\left(-\epsilon w_{k i}\right)+F^{u}\left(-\epsilon w_{k}\right)+F^{x_{k}}+\epsilon\left(w_{t k}-(\mathbb{B} w)_{x_{k}}\right),
\end{gathered}
$$

other derivatives can be calculated in a similar way. Substituting these into $Q^{*}=$ $Q_{m}^{*}(\epsilon)$ defined in $(2.5)$, we get

$$
\begin{aligned}
Q^{*}= & \sum_{i, j, k, l=1}^{n} F_{\epsilon}^{i j, k l} X_{i j} X_{k l}+2 \sum_{i, j, k, l=1}^{n} F_{\epsilon}^{i j}\left(Q \tilde{A} Q^{T}\right)_{k l} X_{i k} X_{j l}-2 \sum_{i, j=1}^{n} F_{\epsilon}^{i j, u} X_{i j} Y \\
& -2 \sum_{i, j, k=1}^{n} F_{\epsilon}^{i j, x_{k}} X_{i j} z_{k}+F_{\epsilon}^{u, u} Y^{2}+2 \sum_{i=1}^{n} F_{\epsilon}^{u, x_{i}} Y z_{i}+\sum_{i, j=1}^{n} F_{\epsilon}^{x_{i}, x_{j}} z_{i} z_{j} \\
(3.3) \quad= & I_{1}+I_{2}+I_{3},
\end{aligned}
$$


where $F_{\epsilon}^{i j, k l}, F_{\epsilon}^{i j}, F_{\epsilon}^{u, u}, F_{\epsilon}^{i j, u}, F_{\epsilon}^{i j, x_{k}}, F_{\epsilon}^{u, x_{i}}, F_{\epsilon}^{x_{i}, x_{j}} \quad$ are $\quad$ evaluated at $\left(Q \tilde{B} Q^{T}, \nabla u(x, t), u, x, t\right)$ and

$$
\begin{aligned}
I_{1}= & \sum_{i, j, k, l=1}^{n} F^{i j, k l}\left(X_{i j}+\epsilon w_{z i j}\right)\left(X_{k l}+\epsilon w_{z k l}\right) \\
& +2 \sum_{i, j, k=1}^{n} F^{i j, p_{k}}\left(X_{i j}+\epsilon w_{z i j}\right)\left(\epsilon w_{z k}\right)+2 \sum_{i, j=1}^{n} F^{i j, u}\left(X_{i j}+\epsilon w_{z i j}\right)\left(-Y+\epsilon w_{z}\right) \\
& +2 \sum_{i, j, k=1}^{n} F^{i j, x_{k}}\left(X_{i j}+\epsilon w_{z i j}\right)\left(-z_{k}\right)+\sum_{k, l=1}^{n} F^{p_{k}, p_{l}}\left(\epsilon w_{z k}\right)\left(\epsilon w_{z l}\right) \\
& +2 \sum_{k}^{n} F^{p_{k}, u}\left(\epsilon w_{z k}\right)\left(-Y+\epsilon w_{z}\right)+2 \sum_{k, i=1}^{n} F^{p_{k}, x_{i}}\left(\epsilon w_{z k}\right)\left(-z_{i}\right)+F^{u, u}\left(-Y+\epsilon w_{z}\right)^{2} \\
& +2 \sum_{i=1}^{n} F^{u, x_{i}}\left(-Y+\epsilon w_{z}\right)\left(-z_{i}\right)+\sum_{i, j=1}^{n} F^{x_{i}, x_{j}}\left(-z_{i}\right)\left(-z_{j}\right), \\
I_{2}= & 2 \sum_{i, j, k, l=1}^{n} F^{i j}\left(Q \tilde{A} Q^{T}\right)_{k l} X_{i k} X_{j l}, \\
I_{3}= & \epsilon\left(w_{t z z}-\sum_{i, j}^{n} F^{i j} w_{z z i j}-\sum_{k=1}^{n} F^{p_{k}} w_{z z k}-F^{u} w_{z z}-\left(\mathbb{B} w_{z z}\right),\right.
\end{aligned}
$$

where functions $F^{i j, k l}, F^{i j}, F^{u, u}, F^{i j, u}, F^{i j, x_{k}}, F^{u, x_{i}}, F^{x_{i}, x_{j}}$ are assumed their values at the point $\left(Q \tilde{B} Q^{T}-\epsilon \nabla^{2} w, p-\epsilon \nabla w, u-\epsilon w, x, t\right)$. It follows that from conditions (1.9) and (1.6)

$$
I_{1} \geq 0, \quad I_{2} \geq 0
$$

Since for $|z|=1$,

$$
g_{z z} \geq\left(1+|x|^{2}\right)^{\frac{m}{2}+\alpha}
$$

and

$$
\left|\nabla^{2} g\right|+(1+|x|)\left|\nabla^{3} g\right|+\left(1+|x|^{2}\right)\left|\nabla^{4} g\right| \leq C\left(1+|x|^{2}\right)^{\frac{m}{2}+\alpha} .
$$

We have, from condition (1.8)

$$
(\mathbb{B} g)_{z z}=O\left(\left(1+|x|^{2}\right)^{\frac{m}{2}+\alpha}\right)
$$

and from condition (1.10)

$$
\sum_{i, j}^{n}\left|F^{i j} g_{z z i j}\right|+\sum_{k=1}^{n}\left|F^{p_{k}} g_{z z k}\right|+F^{u} g_{z z} \leq C\left(1+|x|^{2}\right)^{\frac{m}{2}+\alpha} .
$$

We consequently obtain

$$
\begin{aligned}
& w_{t z z}-\sum_{i, j}^{n} F^{i j} w_{z z i j}-\sum_{k=1}^{n} F^{p_{k}} w_{z z k}-F^{u} w_{z z}-(\mathbb{B} w)_{z z} \\
= & e^{K t}\left(K g_{z z}-\sum_{i, j}^{n} F^{i j} g_{z z i j}-\sum_{k=1}^{n} F^{p_{k}} g_{z z k}-F^{u} g_{z z}-(\mathbb{B} g)_{z z}\right) \geq 0
\end{aligned}
$$


if $K$ is large enough. This implies $I_{3} \geq 0$ and the lemma is proved. This completes the proof of Theorem 1.1.

REMARK 3.2. As we mentioned that most of the conditions imposed in Theorem 1.1 are for the purpose of constructing certain barrier at $\infty$. For example equation $(1.5)$

$$
\mathbb{B} u=\lambda(t) \int_{-\infty}^{\infty} u(x+\eta, t) p(\eta) d \eta
$$

Theorem 1.1 is true with conditions (1.7) and (1.8) replaced by

$$
\int_{-\infty}^{\infty}(1+|\eta|)^{m} p(\eta) d \eta<\infty, \quad \forall m>0 .
$$

It is clear that the result in Theorem 1.1 can be proved under weaker conditions using the same arguments in the proof, but conditions in Theorem 1.1 are simpler though not as general.

4. Parabolic Bellman Equations. In this section, we consider parabolic Bellman equations

$$
u_{t}=\sup _{\alpha \in \mathcal{A}}\left\{\mathcal{L}^{\alpha} u+f^{\alpha}\right\}+\mathbb{B} u, \quad(x, t) \in \mathbb{R}^{n} \times[0, T],
$$

where index set $\mathcal{A}=\{1,2, \cdots\}$ and $\mathcal{L}^{\alpha}$ is a linear operator

$$
\mathcal{L}^{\alpha} u=a_{i j}^{\alpha} u_{i j}+b_{i}^{\alpha} u_{i}+c^{\alpha} u .
$$

Assume that $a_{i j}^{\alpha}, f^{\alpha} \in C^{3,0}\left(\mathbb{R}^{n} \times[0, T]\right), \mathcal{L}^{\alpha}$ is elliptic in the sense

$$
a_{i j}^{\alpha}(x, t) \xi_{i} \xi_{j} \geq \lambda|\xi|^{2}, \quad \forall(x, t) \in \mathbb{R}^{n} \times[0, T], \xi \in \mathbb{R}^{n}
$$

$\lambda>0$ and there is a constant $M$ such that

$$
\left|a_{i j}^{\alpha}(x, t)\right| \leq M\left(1+|x|^{2}\right),\left|\nabla_{x} a_{i j}^{\alpha}(x, t)\right| \leq M(1+|x|)
$$

for all $\alpha \in \mathcal{A}$. We assume that $n=1$ or $n \geq 2$ and

$$
\left(\nabla_{z z}^{2} a_{i j}^{\alpha}\right)\left(Q \tilde{B} Q^{T}\right)_{i j}+2 \sum_{i, j=1}^{n}\left(\nabla_{z} a_{i j}^{\alpha}\right) X_{i j}+2 \sum_{i, j, k, l=1}^{n} a_{i j}^{\alpha}\left(Q \tilde{A} Q^{T}\right)_{k l} X_{i k} X_{j l} \geq 0
$$

for any fixed $0 \leq l \leq n-1, \tilde{B}, \tilde{A}, Q \in \mathbb{O}_{n}, \tilde{X}=(X, Y, z) \in \mathcal{S}_{l}(Q) \times \mathbb{R} \times \mathbb{R}^{n}$ and $z^{m}=\left(Q_{1 m}, \cdots, Q_{n m}\right), m=1, \cdots, n-l$. In addition, suppose

$$
b_{i}^{\alpha}(x, t)=b_{i j}^{\alpha}(t) x_{j}, c^{\alpha}(x, t)=c^{\alpha}(t), b_{i j}^{\alpha}, c^{\alpha} \in C[0, T] .
$$

and $b_{i j}^{\alpha}, c^{\alpha}$ are bounded uniformly.

Theorem 4.1. Assume (1.7) and (1.8) hold. Suppose $f^{\alpha}$ is convex and conditions (4.2), (4.3), (4.4), (4.5) hold. If $u \in C^{4,2}\left(\mathbb{R}^{n} \times[0, T]\right)$ is a solution of (4.1), $\nabla^{2} u \in C_{\text {pol }}$, then $u(x, t)$ is convex provided the initial date $u(x, 0)$ is convex.

Proof. The proof is similar to the proof of Theorem 1.1, we only indicate some necessary modifications. Since Bellman operator in (4.1) is not smooth in its arguments, we first need to mollify it as in [10]. We will apply our analysis in the previous 
section on mollified operators to obtain convexity of the solution to these modified equations. The convexity of the original solution would follow directly by taking the limit.

Let

$$
G_{0}^{k}(y)=\max \left\{y_{1}, \cdots, y_{k}\right\}
$$

and

$$
G_{h}^{k}(y)=h^{-k} \int_{\mathbb{R}^{k}} \rho\left(\frac{y-z}{h}\right) G_{0}^{k}(z) d z
$$

where $\rho$ is a mollifier on $\mathbb{R}^{k}$. Since $G_{0}^{k}(y)$ is convex, it follows that $G_{h}^{k}(y)$ is also convex. Furthermore we have

$$
0 \leq \nabla_{\alpha} G_{h}^{k}(y) \leq 1, \quad \sum_{\alpha=1}^{k} \nabla_{\alpha} G_{h}^{k}(y)=1 .
$$

The convexity of solution of Bellman equation (4.1) can be treated by approximation of solution $u_{h, k}$ of equation

$$
u_{t}=F_{h, k}\left(\nabla^{2} u, \nabla u, u, x, t\right)+\mathbb{B} u,
$$

where

$$
F_{h, k}(r, p, u, x, t)=G_{h}^{k}\left(\mathcal{L}^{1} u+f^{1}, \cdots, \mathcal{L}^{k} u+f^{k}\right) .
$$

In the rest of this section, we will write $G$ for $G_{h}^{k}, F$ for $F_{h, k}$ and $u$ for $u_{h, k}$.

We use the same notations as in the proof of Theorem 1.1. As before, we set $F_{\epsilon}$ as in equation (3.2), check conditions on $F_{\epsilon}$ and apply Theorem 1.2. It is clear that $F_{\epsilon}$ is elliptic. The remainder is to check (2.6) for any fixed $0 \leq l \leq n-1, \tilde{B}, \tilde{A}, Q \in \mathbb{O}_{n}$, $\tilde{X^{m}}=\left(X, Y, z^{m}\right) \in \mathcal{S}_{l}(Q) \times \mathbb{R} \times \mathbb{R}^{n}$ and $z^{m}=\left(Q_{1 m}, \cdots, Q_{n m}\right), m=1, \cdots, n-l$.

We obtain by computation

$$
\begin{aligned}
& F^{i j}=G_{\alpha} a_{i j}^{\alpha}, F^{p_{i}}=G_{\alpha} b_{i}^{\alpha}, F^{u}=G_{\alpha} c^{\alpha}, F^{x_{k}}=G_{\alpha}\left(a_{i j}^{\alpha} r_{i j}+b_{i}^{\alpha} p_{i}+c^{\alpha} u+f^{\alpha}\right)_{x_{k}}, \\
& F^{i j, k l}=G_{\alpha \beta} a_{i j}^{\alpha} a_{k l}^{\beta}, F^{i j, p_{k}}=G_{\alpha \beta} a_{i j}^{\alpha} b_{k}^{\beta}, F^{i j, u}=G_{\alpha \beta} a_{i j}^{\alpha} c^{\beta}, \\
& F^{i j, x_{k}}=G_{\alpha \beta} a_{i j}^{\alpha}\left(a_{i j}^{\alpha} r_{i j}+b_{i}^{\alpha} p_{i}+c^{\alpha} u+f^{\alpha}\right)_{x_{k}}+G_{\alpha}\left(a_{i j}^{\alpha}\right)_{x_{k}},
\end{aligned}
$$

other derivatives can be calculated in a similar way.

Substitute the above into (3.3) and divide $Q^{*}=Q_{m}^{*}(\epsilon)=J_{1}+J_{2}+J_{3}$ as follows

$$
\begin{aligned}
J_{1}= & \sum_{\alpha, \beta} G_{\alpha \beta}\left\{a_{i j}^{\alpha}\left(X_{i j}+\epsilon w_{z i j}\right)+b_{i}^{\alpha}\left(\epsilon w_{z i}\right)+c^{\alpha}\left(-Y+\epsilon w_{z}\right)\right. \\
& \left.-\left[\left(\nabla_{z} a_{i j}^{\alpha}\right)\left(Q \tilde{B} Q^{T}\right)_{i j}+\left(\nabla_{z} b_{i}^{\alpha}\right) u_{i}+\left(\nabla_{z} c^{\alpha}\right) u+\nabla_{z} f^{\alpha}\right]\right\} \times \\
& \left\{a_{i j}^{\beta}\left(X_{i j}+\epsilon w_{z i j}\right)+b_{i}^{\beta}\left(\epsilon w_{z i}\right)+c^{\beta}\left(-Y+\epsilon w_{z}\right)\right. \\
& \left.-\left[\left(\nabla_{z} a_{i j}^{\beta}\right)\left(Q \tilde{B} Q^{T}\right)_{i j}+\left(\nabla_{z} b_{i}^{\beta}\right) u_{i}+\left(\nabla_{z} c^{\beta}\right) u+\nabla_{z} f^{\beta}\right]\right\}, \\
J_{2}= & \sum_{\alpha} G_{\alpha}\left[\left(\nabla_{z z} a_{i j}^{\alpha}\right)\left(Q \tilde{B} Q^{T}\right)_{i j}+\left(\nabla_{z z} b_{i}^{\alpha}\right) u_{i}+\left(\nabla_{z z} c^{\alpha}\right) u+\nabla_{z z} f^{\alpha}-2\left(\nabla_{z} a_{i j}^{\alpha}\right) X_{i j}\right. \\
& \left.+2\left(\nabla_{z} c^{\alpha}\right)\left(Y-\epsilon w_{z}\right)+2 a_{i j}^{\alpha}\left(Q \tilde{A} Q^{T}\right)_{k l} X_{i k} X_{j l}\right], \\
J_{3}= & \epsilon\left[w_{t z z}-(\mathbb{B} w)_{z z}-\sum_{\alpha} G_{\alpha}\left(a_{i j}^{\alpha} w_{z z i j}+b_{i}^{\alpha} w_{z z i}+c^{\alpha} w_{z z}+2\left(\nabla_{z} a_{i j}^{\alpha}\right) w_{z i j}+\left(\nabla_{z} b_{k}^{\alpha}\right) w_{z k}\right]\right.
\end{aligned}
$$


Following the same lines of proof in the last section, we only need to check $J_{i} \geq 0$ for $i=1,2,3$. By the convexity of $G(y), J_{1} \geq 0$. From (4.5), we deduce

$$
J_{2}=G_{\alpha}\left[\left(\nabla_{z z}^{2} a_{i j}^{\alpha}\right)\left(Q \tilde{B} Q^{T}\right)_{i j}-2\left(\nabla_{z} a_{i j}^{\alpha}\right) X_{i j}+2 a_{i j}^{\alpha}\left(Q \tilde{A} Q^{T}\right)_{k l} X_{i k} X_{j l}+\nabla_{z z}^{2} f^{\alpha}\right] .
$$

Consequently, $J_{2} \geq 0$ by (4.4)(note that $X$ can be replaced by $-X$ in (4.4)) and the convexity of $f^{\alpha}$. As in the previous section, by (4.6), (4.3), (4.5), (1.7) and (1.8), we obtain

$$
K g_{z z}-(\mathbb{B} g)_{z z}-G_{\alpha}\left(a_{i j}^{\alpha} g_{z z i j}+b_{k}^{\alpha} g_{z z k}+c^{\alpha} g_{z z}+2\left(\nabla_{z} a_{i j}^{\alpha}\right) g_{z i j}+2\left(\nabla_{z} b_{k}^{\alpha}\right) g_{z k}\right] \geq 0
$$

if $K$ is large enough. Hence $J_{3} \geq 0$. This completes the proof. $\square$

REMARK 4.2. Theorem 4.1 is still true if the index set $\mathcal{A}$ is a separable metric space. Moreover, under similar conditions, we can also get the convexity preserving for following equation

$$
u_{t}=\sup _{\alpha \in \mathcal{A}}\left\{\mathcal{L}^{\alpha} u+f^{\alpha}+\mathbb{B}^{\alpha} u\right\}, \quad(x, t) \in \mathbb{R}^{n} \times[0, T]
$$

where

$$
\mathbb{B}^{\alpha} u=\lambda^{\alpha}(t) \int_{0}^{1}\left(u\left(x+\psi^{\alpha}(x, t, \eta), t\right)-u(x, t)-\psi^{\alpha}(x, t, \eta) \cdot \nabla u(x, t)\right) d \eta .
$$

\section{REFERENCES}

[1] O. Alvarez, J.M. Lasry and P.-L. Lions, Convexity viscosity solutions and state constraints, J. Math. Pures Appl., 76 (1997), pp. 265-288.

[2] F.E. Benth, K.H. Karlsen, and K. Reikvam, Optimal portfolio selection with consumption and nonlinear integro-differential equations with gradient constraint: A viscosity solution approach , Finance and Stochastics, 5 (2001), pp. 275-303.

[3] F.E. Benth, K.H. Karlsen, and K. Reikvam, Optimal portfolio management rules in a nonGaussian market with durability and intertemporal substitution, Finance and Stochastics, 5 (2001), pp. 447-467.

[4] B. Bian And P. Guan, A microscopic convexity principle for fully nonlinear differential equations, preprint.

[5] L. Caffarelli, P. Guan and X. Ma, A constant rank theorem for solutions of fully nonlinear elliptic equations, Communications on Pure and Applied Mathematics, 60 (2007), pp. 17691791 .

[6] R. Cont And P. Tonkov, Financial modelling with jump processes, Chapman and Hall/CRC, 2004.

[7] E. Ekstrom And J. Tysk, properties of option prices in models with jumps, Mathematical Finance, 17 (2007), pp. 381-397.

[8] E. Ekstrom and J. Tysk, Convexity preserving jump diffusion models for option pricing, J. Math. Anal. Appl., 330 (2007), pp. 715-728.

[9] Y. Giga, S. Goto, H. Ishi And M.H. Sato, Comparison principle and convexity preserving properties for singular degenerate parabolic equations on unbounded domains, Indiana Univ. Math. J., 40 (1991), pp. 443-470.

[10] D. Gilbarg And N.S. Trudinger, Elliptic partial differential equations of second order, Springer 1997.

[11] P. Guan And X.N. Ma, The Christoffel-Minkowski Problem I: Convexity of Solutions of a Hessian Equations, Inventiones Math., 151 (2003), pp. 553-577.

[12] S. JANSON AND J. TYSK, Preservation of convexity of solutions to parabolic equations, J. Differential Equations, 206 (2004), pp. 182-226.

[13] S. LENHART, Integro-differential operators associated with diffusion processes with jumps, Appl. Math. Optim., 9 (1982), pp. 177-191. 
[14] R.C. Merton, Continuous time finance, Blackwell Publishers, 1994.

[15] M. Romano and N. Touzi, Contingent claims and market completeness in a stochastic volatility model, Mathematical Finance, 7 (1997), pp. 399-410.

[16] H.M. Soner, Optimal control of jump-Markov processes and viscosity solutions, In: Stochastic differential systems, stochastic control theory and applications, IMA Vol. Math. APPL., 10, New York: Spring 1988, pp. 501-511.

[17] S.E. Shreve And H.M. Soner, Optimal investment and consumption with transaction costs, The Annals of Appl. Prob., 4 (1994), pp. 609-692.

[18] H.M. Soner And N. TouzI, Super-replication under gamma constraints, Siam J. Control and Opt., 39 (2002), pp. 73-96.

[19] C. YAng, L. Jiang AND B. Bian, Free boundary and American options in a jump-diffusion model, Euro. Jnl of Applied Mathematics, 17 (2006), pp. 95-127. 
B. BIAN AND P. GUAN 